\title{
Suplementación con plasma seminal y relación de sus componentes con la calidad de semen congelado- descongelado de asnos (Equus asinus)
}

\author{
Supplementation with seminal plasma and relationship of its \\ components with the quality of frozen-thawed semen of \\ donkeys (Equus asinus)
}

\author{
Juan David Montoya Páez ${ }^{a}$, Benjamín Alberto Rojanob ${ }^{b}$ Giovanni Restrepo Betancurc*
}

\begin{abstract}
RESUMEN
El objetivo fue evaluar la suplementación con plasma seminal y la relación existente entre algunos de sus componentes, con la calidad del semen congelado-descongelado de asnos (Equus asinus). El semen de cinco asnos criollos colombianos se colectó por el método de la vagina artificial. A partir de una fracción de cada eyaculado se separó el plasma seminal por centrifugación y se evaluó su contenido de vitamina C (VC), vitamina E (VE), proteínas totales (PT) y perfil lipídico (PL). La criopreservación del semen se realizó por congelación convencional en un diluyente suplementado con $\mathbf{2 0} \%$ de plasma seminal. Se evaluó la movilidad, la integridad estructural de membrana (IEM), la morfología anormal (MA) y la integridad funcional de membrana (HOS) de los espermatozoides, mediante el sistema computarizado SCA ${ }^{\circledR}$, el ensayo fluorescente SYBR14/IP, la tinción con eosina-nigrosina y la prueba hipoosmótica, respectivamente. Se ajustaron modelos mixtos y se realizó un análisis de correlación de Pearson entre los componentes del plasma seminal y la calidad espermática. Se encontró que un nivel alto de VC, VE, PT y ácido esteárico (C18:0) tuvo un efecto deletéreo sobre la calidad del semen criopreservado $(P \leq 0.05)$. Mientras que un nivel bajo de PT y C18:0, y un nivel medio de VE y VC presentaron resultados post-descongelación superiores para la movilidad y la integridad de membrana $(P \leq 0.05)$. Se hallaron coeficientes de correlación negativos de VC, VE, PT y C18:0 con diferentes parámetros de calidad seminal post-descongelación. Se concluye que la composición del plasma seminal suplementado para la congelación de semen de asno, influye en la calidad espermática post-descongelación.
\end{abstract}

PALABRAS CLAVE: Criopreservación, Semen de asno, Lípidos, Proteínas, Vitaminas.

\section{ABSTRACT}

The objective was to evaluate seminal plasma supplementation and the relationship between some of its components with frozen-thawed semen quality of donkey (Equus asinus). Semen from five Colombian Creole donkeys was collected by the artificial vagina method. From a fraction of each ejaculate the seminal plasma was separated by centrifugation, and vitamin C (VC), vitamin E (VE), total protein (TP) and lipid profile (LP) was evaluated. Semen cryopreservation was performed by conventional freezing in supplemented extender with $20 \%$ of seminal plasma. Motility, structural membrane integrity (SMI), abnormal morphology (AM) and functional membrane integrity (HOS) of sperm, was assessed by SCA ${ }^{\circledR}$ computerized system, SYBR14 / IP fluorescent assay, eosin-nigrosine staining and hypo osmotic swelling test, respectively. Mixed models were fitted where the fixed effect of the level of each component is included and a Pearson correlation analysis was performed between the plasma components and sperm quality. Means were compared by the Tukey test. It was found that high levels of VC, VE, TP and stearic acid (C18:0) had a deleterious effect on cryopreserved semen quality $(P \leq 0.05)$. While a low level of PT and C18: 0 , and a medium level of VE and VC showed higher post-thaw results for motility and membrane integrity $(P \leq 0.05)$. Negative correlation coefficients of VC, VE, TP and C18:0 with different parameters of post-thaw semen quality were found. It is concluded that the composition of seminal plasma supplemented in freezing of donkey semen, influences the post-thaw sperm quality.

KEY WORDS: Cryopreservation, Donkey semen, Lipids, Proteins, Vitamins.

Recibido el 23 de febrero de 2016. Aceptado el 21 de julio de 2016.

a Facultad de Ciencias Agrarias, Politécnico Colombiano Jaime Isaza Cadavid. Carrera 48 No 7-151. Medellín, Colombia.

${ }^{b}$ Facultad de Ciencias, Universidad Nacional de Colombia Sede Medellín. Medellín, Colombia.

c Facultad de Ciencias Agrarias, Universidad Nacional de Colombia Sede Medellín. Medellín, Colombia.

*Autor de correspondencia: grestre0@unal.edu.co. 


\section{INTRODUCCIÓN}

La población de asnos (Equus asinus) se ha incrementado ampliamente a lo largo de las últimas décadas en América del Sur, dado que su interés radica en la producción de mulares para el deporte, el ocio y sobre todo para el manejo de los rebaños de ganado vacuno ${ }^{(1)}$. La inseminación artificial con semen criopreservado, es considerada como una de las biotecnologías de la reproducción más importantes para incrementar el número de individuos de muchas especies, como también para mejorar la distribución genética y reducir la consanguinidad ${ }^{(2)}$. Sin embargo, la criopreservación del semen ha requerido convencionalmente la centrifugación del eyaculado, con la finalidad de retirar el plasma seminal, al ser considerado perjudicial para los espermatozoides durante el almacenamiento a bajas temperaturas ${ }^{(3,4)}$. En équidos se ha observado que el uso de altas proporciones de dilución de los eyaculados, así como la eliminación parcial o total del plasma seminal, pueden reducir los efectos nocivos de este fluido biológico sobre los espermatozoides ${ }^{(5,6)}$.

El plasma seminal contiene una gran diversidad de componentes, sin embargo su papel fisiológico todavía no se entiende completamente ${ }^{(7)}$. En condiciones naturales, se sabe que está involucrado en varios eventos que preceden la fertilización, como la activación de la movilidad, la acción antimicrobiana, la neutralización de metabolitos y la mediación de la capacitación espermática y de la respuesta inflamatoria uterina postcoital(7,8). En equinos (Equus caballus) se han identificado diversos componentes del plasma seminal, así como su relación con calidad del semen fresco y criopreservado ${ }^{(5,9)}$. Sin embargo, poco se conoce en este sentido, respecto al plasma seminal de los asnos y sus componentes. El objetivo de este trabajo fue evaluar la suplementación con plasma seminal y la relación existente entre algunos de sus componentes, con la calidad del semen congelado-descongelado de asnos.

\section{MATERIAL Y MÉTODOS}

\section{Recolección de semen}

Cinco asnos criollos colombianos (Equus asinus), localizados en el Valle del Aburra, Antioquia
(Colombia), se utilizaron para la recolección de dos eyaculados (fracción espermática) por animal. Los animales estuvieron entre los 4 y 8 años de edad, con fertilidad probada por el nacimiento de crías vivas después de la inseminación artificial de yeguas, condición corporal entre 7 y 8 (escala 1-9) y condiciones similares de manejo en estabulación. El semen se colectó por el método de vagina artificial, con un periodo de descanso sexual de ocho días entre los eyaculados (para cada animal). Una fracción de 5 a $10 \mathrm{ml}$ del eyaculado se utilizó para extraer el plasma seminal, mediante centrifugación a $850 \times g$ durante $15 \mathrm{~min}$. El resto del eyaculado se diluyó en proporción 1:1 en EquiPlus ${ }^{\circledR}$ (Minitube, Alemania) y se transportó en refrigeración a $5{ }^{\circ} \mathrm{C}$ (Equitainer ${ }^{\circledR}$, Hamilton Research Inc, USA).

\section{Evaluación de componentes del plasma seminal}

Concentración de proteínas totales (PT). Se evaluó por triplicado para cada muestra de plasma seminal, el cambio de color de Coomassie Brilliant Blue G-250 (BIO-RAD, USA) en respuesta a diferentes concentraciones de proteínas (método de Bradford). Se construyó una curva patrón con una solución de albumina sérica bovina (1 $\mathrm{mg} / \mathrm{ml})$ (Sigma-Aldrich, USA). La evaluación se realizó por espectrofotometría (Multiskan ${ }^{\mathrm{TM}}$, Thermo Scientific, USA) a $595 \mathrm{~nm}$, por $5 \mathrm{~min}$ a temperatura ambiente ${ }^{(10)}$.

Vitamina $C(V C)$ y vitamina $E(V E)$. Se realizó la liofilización del plasma seminal mediante un liofilizador FreeZone ${ }^{\circledR}$ Plus de $2.5 \mathrm{~L}$ (Labconco Corp., USA). El contenido de ambas vitaminas se evaluó por triplicado para cada muestra de plasma seminal, mediante HPLC con un cromatógrafo líquido LC20AD (Shimadzu Sci. Inst. USA), equipado con un auto inyector (SIL-20A /HT), un módulo de comunicación (CBM-20A) y un detector de fila de diodos a $245 \mathrm{~nm}$. El plasma liofilizado se diluyó en agua suprapura, antes de la inyección al cromatógrafo. El contenido de VC (ácido ascórbico) se evaluó según un protocolo modificado(11). La cuantificación se llevó a cabo en una columna C-8 de dimensiones $(5 \mu \mathrm{m}) 250 * 4.6$. Como fase móvil se utilizó ácido fórmico $0.1 \%$. El contenido de VE (atocoferol) se evaluó según un protocolo 
modificado ${ }^{(12)}$. La cuantificación se llevó a cabo en una columna LiChrospher RP-18 de dimensiones (5 $\mu \mathrm{m})$ 250*4.5. Como fase móvil se utilizó metanol/diclorometano (85:15, \% v/v). Para ambas lecturas la razón de flujo de la fase móvil fue 0.8 $\mathrm{ml} / \mathrm{min}, 35{ }^{\circ} \mathrm{C}$ y condiciones isocráticas. Para la identificación y cuantificación de los compuestos se construyeron previamente curvas de calibración con ácido ascórbico y a-tocoferol grado HPLC.

Perfil lipídico. Se evaluó por triplicado para cada muestra de plasma seminal, mediante un cromatógrafo de gases 6890N (Agilent Tech, USA) acoplado a un detector selectivo de espectrofotometría de masas MS 5973N (Agilent Tech, USA) y equipado con un inyector split/splitless. La temperatura del inyector fue $300^{\circ} \mathrm{C}$ y la muestra previamente derivatizada con $\mathrm{KOH}$-metanol se inyectó automáticamente en el modo Split-less. Se usó una columna HP-5 ms (5 \% fenilmetilsiloxano) de $30 \mathrm{~m}, 0.25 \mathrm{~mm}$ con un espesor de película 0.25 $\mu \mathrm{m}$ y una temperatura máxima de $325^{\circ} \mathrm{C}$. El programa de temperatura se inició a $50{ }^{\circ} \mathrm{C}$ hasta $200^{\circ} \mathrm{C}$ (5 min) con una temperatura final de $300^{\circ} \mathrm{C}$ (14 min) a una tasa de $10^{\circ} \mathrm{C} / \mathrm{min}$. Se usó helio como gas portador a un flujo constante de $1 \mathrm{ml} / \mathrm{min}$. La temperatura del detector fue de $300{ }^{\circ} \mathrm{C}$. Para el análisis se utilizó el software MSD ChemStation D 02.00.275 (Agilent Tech, USA) y la base de datos NIST $2005^{(13)}$.

\section{Criopreservación de semen}

Se realizó por medio de un protocolo de congelación modificado en cuanto al diluyente utilizado y el tiempo de exposición a vapores ${ }^{(14)}$. El semen se centrifugó por 12 min a 850 xg y el precipitado se resuspendió en diluyente EquiPlus ${ }^{\circledR}$ (Minitube, Alemania) suplementado con $3 \%$ de glicerol (\% V/V), $5 \%$ de yema de huevo (\% V/V) y $20 \%$ de plasma seminal ( $\% \mathrm{~V} / \mathrm{V}$ ). El plasma seminal utilizado para cada proceso provino del mismo asno y eyaculado procesado. La dilución se realizó hasta alcanzar una concentración final de $100 \times 10^{6}$ espermatozoides $/ \mathrm{ml}$. El semen se mantuvo a $5{ }^{\circ} \mathrm{C}$ por 60 min y se empacó en pajillas de $0.5 \mathrm{ml}$ en un equipo MRS1 Dual V2 (IMV Technologies, Francia). Las pajillas se mantuvieron en refrigeración a $5^{\circ} \mathrm{C}$ durante $1 \mathrm{~h}$ y luego se sometieron a vapores de nitrógeno líquido por 15 min (tasa de enfriamiento aproximada de $-8.3^{\circ} \mathrm{C} / \mathrm{min}$ ) y se almacenaron en un tanque para nitrógeno líquido (tasa de enfriamiento aproximada de $-38^{\circ} \mathrm{C} / \mathrm{seg}$ ). El semen permaneció congelado durante 15 días y se descongeló en agua a $37^{\circ} \mathrm{C}$ por $1 \mathrm{~min}$. Luego se mantuvo temperado a $37^{\circ} \mathrm{C}$ por 5 min antes de la evaluación de la calidad seminal post-descongelación.

\section{Evaluación de la calidad seminal}

Movilidad espermática. Se evaluó mediante el sistema SCA ${ }^{\circledR}$ versión 5.1 (Microptic S.L., España) de acuerdo a un protocolo previamente reportado(15). Se utilizó un microscopio de contraste de fase (Eclipse E200, Nikon, Inc., Japón) con una cámara digital (Scout SCA780, Basler, USA). Se evaluaron los parámetros: movilidad total (MT), movilidad progresiva (MP), velocidad rectilínea (VSL), velocidad curvilínea (VCL), velocidad media (VAP).

Integridad estructural de membrana (IEM). Se evaluó utilizando un procedimiento previamente descrito mediante el kit Live/Dead (Molecular Probes Inc., USA) ${ }^{(16)}$. Se suspendieron $200 \mu$ de la muestra espermática en solución Hanks Heppes con $1 \%$ de albúmina sérica bovina, para una concentración aproximada de $20 \times 10^{6}$ espermatozoides $/ \mathrm{ml}$. Luego la mezcla se incubó a $37^{\circ} \mathrm{C}$ por $8 \mathrm{~min}$, con $6 \mathrm{mM}$ de SYBR14. Seguidamente se incubó de la misma manera, con $0.48 \mathrm{mM}$ de yoduro de propidio. Luego a partir de una muestra de $5 \mu$, se realizó el conteo de 200 espermatozoides, mediante un filtro UV-2A de un microscopio E200 con fluorescencia HBO (Nikon Inc., Japón).

Morfología anormal (MA). Se evaluó mediante la tinción con eosina-nigrosina(17). Sobre un portaobjetos se depositó una gota de muestra y una gota de eosina-nigrosina (Sigma-Aldrich, USA). Ambas gotas se mezclaron y se realizó un extendido, el cual se fijó sobre una platina térmica a $37^{\circ} \mathrm{C}$. En un microscopio de contraste de fase Eclipse E200 (Nikon Inc., Japón), se realizó la evaluación de la morfología de 200 espermatozoides.

Integridad funcional de membrana (HOS). Se evaluó mediante la prueba hipoosmótica ${ }^{(18)}$. Se tomaron $20 \mu \mathrm{l}$ de semen y se adicionaron a un tubo con $200 \mu \mathrm{l}$ de una solución hipoosmótica de sacarosa 
$5.4 \%$ (100 mOsmol/L). Esta mezcla se incubó a $38.5{ }^{\circ} \mathrm{C}$ por $30 \mathrm{~min}$ y luego se evaluó mediante microscopia de contraste de fase (Eclipse E200, Nikon, Inc., USA) la reacción de 200 espermatozoides.

\section{Manejo de información y evaluación estadística}

El control de calidad de los análisis de laboratorio para la recolección de datos experimentales, estuvo sujeto a procedimientos de seguimiento, control, mantenimiento y calibración de equipos, acordes a la normatividad de las entidades participantes. Para los métodos de evaluación de componentes del plasma seminal, las curvas de calibración empleadas se validaron en base a ecuaciones de regresión lineal, con coeficientes de determinación $\left(R^{2}\right)$ superiores a 0.95. Se definió como unidad experimental la pajilla de semen congelado-descongelado. Se realizó el ajuste de modelos mixtos donde se establecieron como variables dependientes, los parámetros de calidad seminal (MT, MP, VCL, VSL, VAP, IEM, MA y HOS). Como variables independientes se incluyeron los efectos del asno y el eyaculado (como un efecto aleatorio anidado) y el efecto fijo del nivel (bajo, medio y alto) de cada componente del plasma seminal. Los rangos de clasificación de los niveles de cada componente se establecieron a partir del análisis descriptivo de la distribución (por cuartiles) de los resultados de los eyaculados evaluados. La normalidad de los datos se aseguró mediante la evaluación por la prueba de Shapiro-Wilk y el uso de transformaciones logarítmicas (logaritmo natural). Se validó el cumplimiento de los supuestos de homogeneidad de varianzas y normalidad de los errores, mediante las pruebas de Bartlett y Kolmogoroff-Smirnoff, respectivamente. Se realizó un análisis de correlación de Pearson entre las variables de calidad seminal y los componentes del plasma seminal. Las comparaciones de medias se realizaron por la prueba de Tukey. Todos los análisis se realizaron con el programa SAS 9.2 (SAS Inst. Inc., Cary, NC).

\section{RESULTADOS Y DISCUSIÓN}

La presencia del plasma seminal durante el almacenamiento del semen a bajas temperaturas, es considerado como perjudicial( ${ }^{(4)}$, dado que puede generar efectos deletéreos sobre la calidad y la vitalidad de los espermatozoides ${ }^{(19)}$. Para la especie equina, se ha descrito que componentes del plasma seminal como vitaminas y enzimas, tienen funciones como antioxidantes ${ }^{(8,9)}$, mientras algunas proteínas cumplen roles importantes en la interacción con el tracto genital o la modulación del sistema inmune de la hembra(20). Así mismo, proteínas como las denominadas proteínas del plasma seminal (HSP) y las proteínas secretoras ricas en cisteína (CRISP), se han relacionado con la fertilidad y la criotolerancia del semen equino(21,22). Respecto a los lípidos del plasma seminal, se conoce que el colesterol y los fosfolípidos tienen la función de prevenir la aparición prematura de la capacitación, a través de la estabilización de la membrana plasmática de los espermatozoides ${ }^{(23)}$.

Los resultados de composición del plasma seminal muestran una amplia variabilidad para la concentración de estas vitaminas entre los asnos evaluados (Cuadro 1), lo cual podría relacionarse con las marcadas diferencias reportadas en la concentración de ácido ascórbico plasmático en otros équidos ${ }^{(24)}$, que al igual que la concentración plasmática del a-tocoferol, está ligada a su consumo 0 administración ${ }^{(25)}$.

Cuadro 1. Componentes del plasma seminal (media \pm desviación estándar) ${ }^{\star}$

\begin{tabular}{lcrrrrrr}
\hline & Media & CV & \multicolumn{1}{c}{ Asno 1 } & \multicolumn{1}{c}{ Asno 2 } & \multicolumn{1}{c}{ Asno 3 } & \multicolumn{1}{c}{ Asno 4 } & \multicolumn{1}{c}{ Asno 5 } \\
\hline PT & $15.4 \pm 1.0$ & 6.8 & $15.3 \pm 0.6$ & $15.5 \pm 0.0$ & $14.6 \pm 1.2$ & $16.85 \pm 0.2$ & $14.8 \pm 0.8$ \\
VC & $163.9 \pm 118.6$ & 72.3 & $38.1 \pm 6.4$ & $175.4 \pm 39.5$ & $124.7 \pm 15.1$ & $345.40 \pm 101.7$ & $136.0 \pm 89.4$ \\
VE & $238.2 \pm 62.2$ & 26.1 & $133.4 \pm 0.0$ & $202.7 \pm 60.0$ & $266.9 \pm 7.0$ & $253.70 \pm 60.6$ & $282.1 \pm 41.8$ \\
\hline
\end{tabular}

*= 10 muestras por componente; $\mathrm{CV}=$ coeficiente de variación (\%); $\mathrm{PT}=$ proteínas totales (mg de BSA equivalente/ml); VC= vitamina C (mg/l); VE= vitamina $\mathrm{E}(\mathrm{mg} / \mathrm{l})$. 
Algo similar sucedió con el perfil lipídico del plasma seminal, para el cual no se observó homogeneidad en los ácidos grasos presentes entre asnos y eyaculados. Tan solo para el ácido esteárico (C18:0), correspondiente al ácido graso con una presencia más consistente en la mayoría de eyaculados, se encontró un coeficiente de variación (CV) de $35.6 \%$. Mientras para ambos eyaculados de uno de los asnos evaluados (asno 3), no se detectó la presencia de ácidos grasos (Cuadro 2). Sin embargo, en este caso es posible considerar limitaciones en el método analítico empleado para la detección de los ácidos grasos y otros factores intrínsecos al reproductor que podrían explicar dicho fenómeno. Un grupo de investigadores demostraron actividad de lipasa en el plasma seminal equino y su relación adversa en la refrigeración del semen de algunos ejemplares ${ }^{(26)}$; aunque estos autores, plantearon la necesidad de caracterizar la naturaleza de la actividad de la lipasa en el plasma seminal, es posible que su actividad de hidrólisis esté involucrada en las variaciones en la composición de los ácidos grasos del plasma seminal de équidos.

En general, se reporta que existe una variación considerable en la composición del plasma seminal entre los sementales, al igual que en la interacción de éste con los espermatozoides ${ }^{(27)}$. Como excepción, en esta investigación el contenido de PT fue bastante similar incluso entre los asnos, con un CV inferior al $10 \%$ (Cuadro 1). No se conocen investigaciones donde se reporte la medición de estos componentes en el plasma seminal de asnos.
En el plasma seminal equino se reportan valores de VC equivalentes a $1.76,1.08,2.05$ y $0.84 \mathrm{mg} / \mathrm{L}$, para el otoño, invierno, primavera y verano, respectivamente ${ }^{(9)}$, mientras otros investigadores encontraron una concentración total de VC de 6.0 $\mathrm{mg} / \mathrm{L}$ en equinos ubicados en el trópico ${ }^{(28)}$. Estos resultados son ampliamente inferiores a los encontrados en esta investigación, lo cual indicaría una marcada influencia de la especie en la concentración de VC del plasma seminal de los équidos. Respecto al contenido de proteínas del plasma seminal, se reportan resultados de proteínas totales por eyaculado en caballos fértiles $y$ subfértiles, de $740.8 \pm 489.6 \mathrm{mg}$ y $416.4 \pm 254.7$ $\mathrm{mg}$, respectivamente ${ }^{(29)}$.

Las muestras colectadas de semen fresco presentaron resultados promedio de volumen (libre de gel) de $45.7 \pm 18.2 \mathrm{ml}$ y concentración de $249 \pm$ $67 \times 10^{6}$ espermatozoides/ml. (Cuadro 3). Todos los asnos presentaron una buena calidad seminal, tomando como referencia reportes previos ${ }^{(30,31)}$. Se conoce que la edad de los reproductores puede ser una fuente de variabilidad en los resultados de calidad seminal; en equinos, un estudio con machos de nueve razas y un rango de edad entre 2 y 26 años, reveló una variación significativa por efecto de la edad, en el volumen, la concentración y la morfología espermática ${ }^{(32)}$. En el presente estudio, aunque el rango de edad fue sólo de 4 años, los parámetros volumen y concentración presentaron igualmente mayor variabilidad (CV: 39.8 y $26.9 \%$, respectivamente), en comparación con los demás

Cuadro 2. Perfil lipídico del plasma seminal de asnos (\%)

\begin{tabular}{cccccccc}
\hline Asno & Eyaculado & C13:0 & C15:0 & C16:0 & C18:0 & C18:1 & C18:2 \\
\hline 1 & 1 & 0 & 0 & 15.16 & 19.46 & 49.29 & 16.09 \\
& 2 & 0 & 57.48 & 0 & 42.52 & 0 & 0 \\
2 & 1 & 73.49 & 0 & 0 & 26.51 & 0 & 0 \\
& 2 & 0 & 64.78 & 0 & 35.22 & 0 & 0 \\
3 & 1 & 0 & 0 & 0 & 0 & 0 & 0 \\
& 2 & 0 & 0 & 0 & 0 & 0 & 0 \\
4 & 1 & 33.44 & 0 & 0 & 14.56 & 39.98 & 12.01 \\
& 2 & 0 & 0 & 79.08 & 20.98 & 0 & 0 \\
5 & 1 & 0 & 0 & 60.71 & 39.29 & 0 & 0 \\
& 2 & 0 & 66.38 & 0 & 24.33 & 0 & 9.29 \\
\hline
\end{tabular}

C13= ácido tridecanoico; $C 15: 0=$ ácido pentadecanoico; $C 16: 0=$ ácido palmítico; $C 18: 0=$ ácido esteárico; $C 18: 1=$ ácido oleico; $C 18: 2=$ ácido linoleico. 
parámetros evaluados ( $\mathrm{CV} \leq 20 \%)$. En este trabajo, en el análisis de modelos mixtos para cada variable de calidad y composición seminal se incluyó el efecto aleatorio del asno, con el fin de reducir la variabilidad por factores inherentes al reproductor, como sería el caso de la edad.

El análisis de correlación entre los componentes del plasma seminal y la calidad del semen fresco, arrojó coeficientes negativos $(P \leq 0.05)$, para el contenido de VC con la MT $(r=-0.703)$ y la MP $(r=$ -0.833). Acorde con lo anterior, investigadores hallaron una mayor concentración de VC en caballos de baja fertilidad, lo cual estuvo relacionado no solo con una reducción de la movilidad, sino también con una menor concentración espermática y una mayor proporción de espermatozoides anormales ${ }^{(9)}$. También en este mismo estudio la suplementación con altas concentraciones de ácido ascórbico, demostró un efecto negativo sobre la peroxidación lipídica de la membrana. Se conoce que en presencia de metales de transición, la VC puede actuar como pro-oxidante, al tornarse en un radical altamente reactivo y más destructivo, y por lo tanto generar más radicales libres ${ }^{(33)}$.

La evaluación post-descongelación de la calidad del semen, denotó diferencias marcadas en la congelabilidad de los espermatozoides, para los diferentes asnos. Lo anterior considerando los resultados de calidad seminal en fresco, donde animales con promedios inferiores para diferentes parámetros de calidad seminal, presentaron resultados superiores post-descongelación y viceversa (Cuadro 4). Son escasos los estudios donde se analice en este sentido, el efecto individual de los reproductores asnales; sin embargo, en equinos se ha descrito la existencia de una alta

Cuadro 3. Calidad del semen fresco de asnos (media \pm desviación estándar)

\begin{tabular}{|c|c|c|c|c|c|c|c|c|}
\hline & $\mathrm{n}$ & Media & $\mathrm{CV}$ & Asno 1 & Asno 2 & Asno 3 & Asno 4 & Asno 5 \\
\hline MT & 10 & $92.2 \pm 7.1$ & 7.7 & $98.8 \pm 0.3$ & $95.5 \pm 0.7$ & $84.6 \pm 5.4$ & $97.9 \pm 0.7$ & $84.2 \pm 2.8$ \\
\hline MP & 10 & $68.2 \pm 12.1$ & 17.7 & $85.5 \pm 0.6$ & $63.9 \pm 10.2$ & $66.8 \pm 9.5$ & $69.5 \pm 5.6$ & $55.3 \pm 10.3$ \\
\hline VCL & 10 & $117.8 \pm 19.5$ & 16.5 & $143.5 \pm 7.5$ & $110.2 \pm 15.1$ & $104.6 \pm 18.9$ & $107.6 \pm 2.2$ & $123.3 \pm 27.1$ \\
\hline VSL & 10 & $49.9 \pm 8.8$ & 17.5 & $54.9 \pm 4.9$ & $51.2 \pm 15.9$ & $48.1 \pm 5.0$ & $40.6 \pm 3.9$ & $54.5 \pm 9.6$ \\
\hline VAP & 10 & $84.5 \pm 17.7$ & 20.9 & $104.0 \pm 13.9$ & $82.5 \pm 19.2$ & $66.8 \pm 11.9$ & $75.0 \pm 5.9$ & $93.8 \pm 17.8$ \\
\hline IEM & 10 & $88.2 \pm 6.5$ & 7.3 & $92.0 \pm 1.4$ & $92.0 \pm 1.4$ & $82.5 \pm 2.1$ & $95.0 \pm 1.4$ & $79.5 \pm 0.7$ \\
\hline MA & 10 & $16.3 \pm 2.9$ & 18.3 & $14.5 \pm 2.1$ & $19.0 \pm 1.4$ & $14.5 \pm 0.7$ & $20.0 \pm 1.4$ & $13.5 \pm 0.7$ \\
\hline $\mathrm{HOS}$ & 10 & $63.9 \pm 6.9$ & 10.8 & $69.5 \pm 4.9$ & $66.0 \pm 5.6$ & $55.0 \pm 4.2$ & $69.5 \pm 2.1$ & $59.5 \pm 4.9$ \\
\hline
\end{tabular}

Cuadro 4. Calidad seminal post-descongelación (media \pm desviación estándar)*

\begin{tabular}{lccccccc}
\hline & Media & CV & Asno 1 & Asno 2 & Asno 3 & Asno 4 & Asno 5 \\
\hline MT & $34.2 \pm 22.6$ & 66.2 & $34.8 \pm 20.8^{\mathrm{b}}$ & $31.5 \pm 22.6^{\mathrm{b}}$ & $52.8 \pm 21.2^{\mathrm{a}}$ & $26.2 \pm 18.0^{\mathrm{b}}$ & $25.5 \pm 19.4^{\mathrm{b}}$ \\
MP & $22.0 \pm 17.3$ & 78.6 & $21.8 \pm 15.5^{\mathrm{b}}$ & $19.7 \pm 15.6^{\mathrm{bc}}$ & $38.3 \pm 19.2^{\mathrm{a}}$ & $14.2 \pm 9.7^{\mathrm{c}}$ & $16.2 \pm 14.3^{\mathrm{bc}}$ \\
VCL & $86.3 \pm 20.8$ & 24.1 & $83.2 \pm 21.1^{\mathrm{b}}$ & $92.6 \pm 17.9^{\mathrm{a}}$ & $92.2 \pm 18.7^{\mathrm{a}}$ & $80.5 \pm 12.5^{\mathrm{b}}$ & $83.2 \pm 28.2^{\mathrm{b}}$ \\
VSL & $62.0 \pm 18.4$ & 29.7 & $61.9 \pm 19.7^{\mathrm{bc}}$ & $67.9 \pm 17.3^{\mathrm{a}}$ & $65.3 \pm 12.9^{\mathrm{ab}}$ & $56.6 \pm 11.5^{\mathrm{c}}$ & $58.7^{\mathrm{b}} \pm 25.5^{\mathrm{bc}}$ \\
VAP & $74.4 \pm 20.9$ & 28.1 & $72.7 \pm 21.7^{\mathrm{ab}}$ & $80.9 \pm 18.3^{\mathrm{a}}$ & $80.4 \pm 17.7^{\mathrm{ab}}$ & $67.9 \pm 12.4^{\mathrm{c}}$ & $70.5^{\mathrm{b}} 28.4^{\mathrm{c}}$ \\
IEM & $34.1 \pm 14.0$ & 41.0 & $32.8 \pm 6.2^{\mathrm{b}}$ & $29.0 \pm 10.4^{\mathrm{a}}$ & $46.7 \pm 10.5^{\mathrm{b}}$ & $31.2 \pm 12.6^{\mathrm{b}}$ & $22.1 \pm 4.8^{\mathrm{b}}$ \\
MA & $15.0 \pm 5.8$ & 39.0 & $14.2 \pm 3.0^{\mathrm{b}}$ & $23.8 \pm 3.6^{\mathrm{a}}$ & $13.7 \pm 2.9^{\mathrm{b}}$ & $12.8^{\mathrm{b}} 5.0^{\mathrm{b}}$ & $10.5 \pm 3.5^{\mathrm{c}}$ \\
HOS & $26.7 \pm 12.2$ & 45.5 & $23.4 \pm 12.1^{\mathrm{b}}$ & $26.4 \pm 10.6^{\mathrm{b}}$ & $39.7 \pm 10.9^{\mathrm{a}}$ & $23.7 \pm 7.4^{\mathrm{b}}$ & $15.7 \pm 3.2^{\mathrm{c}}$ \\
\hline
\end{tabular}

${ }^{*}=320$ pajillas por cada parámetro evaluado; $\mathrm{CV}=$ coeficiente de variación (\%); $\mathrm{MT}=$ movilidad total $(\%) ; \mathrm{MP}=$ movilidad progresiva $(\%) ; \mathrm{VCL}=$ velocidad curvilínea $(\mu \mathrm{m} / \mathrm{seg})$; VSL= velocidad lineal $(\mu \mathrm{m} / \mathrm{seg}) ; \mathrm{VAP}=$ velocidad media $(\mu \mathrm{m} / \mathrm{seg})$; IEM: integridad estructural de membrana $(\%) ; \mathrm{MA}=$ morfología anormal $(\%) ;$ HOS= integridad funcional de membrana (\%).

abc Letras diferentes denotan diferencia entre valores $(P \leq 0.05)$. 
variabilidad entre machos en la "crio-supervivencia" del semen ${ }^{(34)}$. Los parámetros que presentaron una menor variabilidad ( $\mathrm{CV}<30 \%$ ) fueron aquéllos relacionados con la cinética espermática como $\mathrm{VCL}$, VSL y VAP.

Para el perfil lipídico del plasma seminal sólo se incluyó el ácido esteárico (C18:0), dado que fue el único ácido graso presente en la mayoría de los asnos evaluados. Se encontró que una alta concentración de VC en el plasma seminal suplementado, tuvo un efecto deletéreo postdescongelación sobre la MT y la MP (Cuadro 5), al igual que se hallaron correlaciones negativas $(P \leq 0.05)$ de la VC con estos mismos parámetros (Cuadro 6). Sin embargo, se observó menor MA en muestras suplementadas con plasma con un nivel

Cuadro 5. Calidad seminal post-descongelación por niveles de componentes (media \pm desviación estándar)

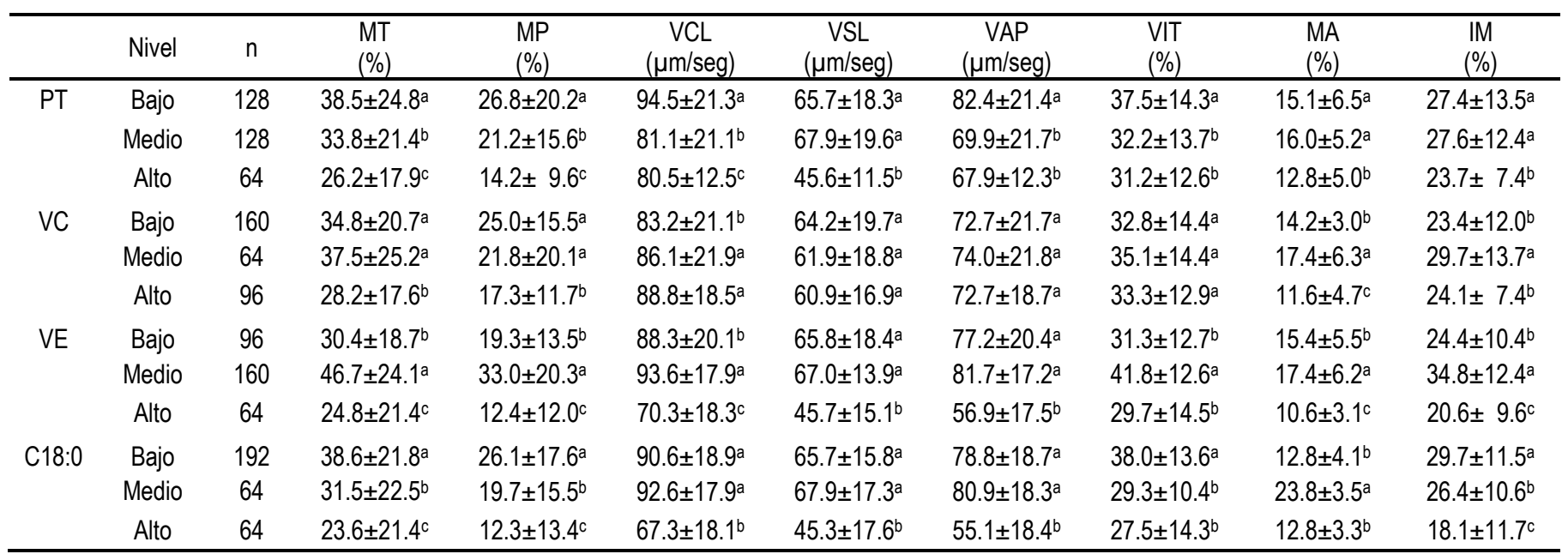

$\mathrm{n}=$ número de pajillas de semen evaluadas; $\mathrm{PT}=$ proteínas totales; $\mathrm{VC}=$ vitamina $\mathrm{C} ; \mathrm{VE}=$ vitamina $\mathrm{E} ; \mathrm{C} 18: 0=$ ácido esteárico; $\mathrm{MT}=$ movilidad total $(\%) ; \mathrm{MP}=$ movilidad progresiva $(\%) ; \mathrm{VCL}=$ velocidad curvilínea $(\mu \mathrm{m} / \mathrm{seg}) ; \mathrm{VSL}=$ velocidad lineal $(\mu \mathrm{m} / \mathrm{seg})=\mathrm{VAP}=$ velocidad media $(\mu \mathrm{m} / \mathrm{seg}) ; \mathrm{IEM}=$ integridad estructural de membrana $(\%) ; \mathrm{MA}=\mathrm{morfología}$ anormal (\%); HOS= integridad funcional de membrana (\%).

Niveles de componentes para PT (mg de BSA equivalente/ml): Bajo < 15.5, Medio 15.5 - 15.8, Alto > 15.8; VITC (mg/l): Bajo < 52.4, Medio 52.4 - 219.6, Alto > 219.6; VITE (mg/l): Bajo < 260.4, Medio 260.4 - 273.5, Alto > 273.5; C18:0 (\%): Bajo < 25.4, Medio 24.5 - 37.2, Alto > 37.2.

abc Letras diferentes (columnas por componente) denotan diferencia $(P \leq 0.05)$.

Cuadro 6. Coeficientes de correlación entre la composición del plasma seminal y la calidad espermática post-descongelación

\begin{tabular}{|c|c|c|c|c|}
\hline & PT & VC & VE & C18:0 \\
\hline MT & $-0.264^{*}(320)$ & $-0.134^{*}(320)$ & $0.082(288)$ & $-0.073(256)$ \\
\hline MP & $-0.354^{*}(320)$ & $-0.125^{*}(320)$ & $0.086(288)$ & $-0.113(256)$ \\
\hline VCL & $-0.308^{*}(320)$ & $0.119^{*}(320)$ & $-0.054(288)$ & $-0.263^{*}(256)$ \\
\hline VSL & $-0.263^{*}(320)$ & $0.115^{*}(320)$ & $-0.146^{*}(288)$ & $-0.269^{*}(256)$ \\
\hline VAP & $-0.302^{*}(320)$ & $0.101(320)$ & $-0.093(288)$ & $-0.267^{*}(256)$ \\
\hline VIT & $-0.289^{*}(320)$ & $-0.066(320)$ & $0.152^{*}(288)$ & $-0.094(256)$ \\
\hline MA & $0.159^{*}(320)$ & $0.071(320)$ & $-0.330^{*}(288)$ & $0.090(256)$ \\
\hline IM & $-0.192^{*}(320)$ & $-0.043(320)$ & $0.065(288)$ & $-0.203^{*}(256)$ \\
\hline
\end{tabular}

$\mathrm{PT}=$ proteínas totales; $\mathrm{VC}=$ vitamina $\mathrm{C} ; \mathrm{VE}=$ vitamina $\mathrm{E} ; \mathrm{C} 18: 0=$ ácido esteárico $\mathrm{MT}=$ movilidad total $(\%) ; \mathrm{MP}=$ movilidad progresiva $(\%)$; VCL= velocidad curvilínea $(\mu \mathrm{m} / \mathrm{seg}) ; \mathrm{VSL}=$ velocidad lineal $(\mu \mathrm{m} / \mathrm{seg}) ; \mathrm{VAP}=$ velocidad media $(\mu \mathrm{m} / \mathrm{seg}) ; \mathrm{VIT}=$ integridad estructural de membrana (\%); MA= morfología anormal (\%); IM= integridad funcional de membrana (\%).

$(n)=$ número de pares de observaciones.

*Coeficientes de correlación significativos $(P \leq 0.05)$. 
alto de esta vitamina. Otros autores han reportado una relación negativa entre la concentración de VC y la calidad del semen equino $0^{(9,28)}$, la cual sería explicada por un efecto pro-oxidante y por lo tanto generador de radicales libres, del ácido ascórbico en presencia de metales de transición ${ }^{(33)}$, sumado al incremento en la generación de ERO, atribuido a los efectos osmóticos y tóxicos de la criopreservación ${ }^{(35)}$.

Se observó que un nivel medio de VE en el plasma seminal suplementado al diluyente, confiere mejores resultados en la calidad del semen descongelado, lo cual fue evidente no solo en la movilidad y cinética espermática, sino también en la integridad $y$ funcionalidad de la membrana plasmática (Cuadro 5). Sin embargo, los coeficientes de correlación entre la VE y los parámetros de calidad seminal, no fueron significativos $(P \geq 0.05)$ (Cuadro 6). Es limitada la información disponible sobre el efecto de la $\mathrm{VE}$, en el semen de asnos. En un estudio donde se incluyó a-tocoferol en la dieta de burros, no se observó efecto sobre la calidad seminal(36). En una investigación reciente donde se evaluó la adición de a-tocoferol en diluyentes para semen equino, no se encontró efecto sobre la calidad seminal(37); sin embargo, otros autores reportaron un efecto promisorio de esta vitamina, en el mejoramiento de la integridad y la estabilidad de la membrana plasmática ${ }^{(38)}$. En otro estudio, el tocoferol redujo la peroxidación lipídica de semen equino sometido a periodos cortos de incubación ${ }^{(39)}$.

El mecanismo antioxidante del a-tocoferol consta de la donación de su hidrógeno fenólico de los radicales peroxilo, formando radicales tocoferoxilo que, a pesar de ser también radicales, no son reactivos y son incapaces de continuar la reacción oxidativa en cadena. Sin embargo, se ha descrito un efecto pro-oxidante del a-tocoferol en altas concentraciones ${ }^{(40)}$, que podría explicar la reducción obtenida en la calidad seminal postdescongelación, de muestras suplementadas con plasma seminal con un nivel alto de $\mathrm{VE}$, respecto a aquéllas suplementadas con plasma con un nivel medio e incluso bajo de esta molécula.

Un nivel alto de PT en el plasma seminal suplementado al diluyente, coincidió con valores inferiores para diferentes parámetros de calidad del semen criopreservado (Cuadro 5). Es probable que fenómenos como la oxidación proteica descrita en el semen equino, esté relacionada con dicho efecto, toda vez que genera grupos carbonilo que se han asociado con la presencia de defectos severos en la función de los espermatozoides ${ }^{(41)}$. Acorde con lo anterior, se hallaron coeficientes de correlación negativos de PT con la mayoría de parámetros de calidad seminal, así como una correlación positiva con la morfología anormal (Cuadro 6). Otros autores no hallaron efecto del contenido de PT sobre la calidad seminal en caballos fértiles y subfértiles ${ }^{(29)}$.

Pocos reportes presentan resultados de la composición lipídica del semen en los équidos. Investigadores reportaron una concentración total de fosfolípidos en el plasma seminal equino de 28.4 $\pm 20.2 \mu$ moles $/ 100 \mathrm{ml}^{(42)}$. En una investigación se encontró que el ácido graso predominante en la membrana de los espermatozoides equinos es el ácido docosopentanoico (C22: $5 \mathrm{n}-6)$, que representa un $49.9 \pm 8.7 \%$ de todos los ácidos grasos, seguido por el ácido palmítico (C16:0) y el ácido esteárico $(\mathrm{C} 18: 0)^{(43)}$. Como se mencionó, en la presente investigación el C18:0 estuvo presente en la mayoría de los eyaculados, sin embargo en diferentes muestras de plasma seminal se observó un predominio proporcional de otros ácidos grasos como el ácido palmítico (C16:0), el ácido pentadecanoico (C15:0) e incluso el ácido oleico (C18:1) (Cuadro 2). Se observó que un alto nivel de esteárico en el plasma seminal suplementado al diluyente, afecta la movilidad y la cinética de los espermatozoides, así como se hallaron correlaciones negativas con la cinética y la integridad de membrana. Son escasos los estudios que aborden el análisis de la relación entre los ácidos grasos del plasma seminal y la calidad espermática de los équidos; sin embargo, se ha descrito una relación del ácido esteárico del plasma seminal, con los parámetros de movilidad del semen humano antes y después de la congelación ${ }^{(44)}$. De otro lado, la composición de ácidos grasos de la membrana plasmática, se ha estudiado por su valor en el diagnóstico de la congelabilidad del semen equino, donde el incremento en la rigidez de la membrana plasmática se atribuye a los ácidos grasos saturados de cadena larga, mientras los ácidos grasos con poli- 
insaturaciones, le otorgan a la membrana una mayor flexibilidad y fluidez ${ }^{(45)}$.

\section{CONCLUSIONES E IMPLICACIONES}

Existe una asociación negativa entre la concentración de vitamina $\mathrm{C}$ y la calidad del semen de asnos, por lo cual se atribuye un efecto deletéreo a la suplementación del diluyente de congelación, con muestras de plasma seminal con un nivel alto de VC. De igual forma, altos niveles de proteínas y ácido esteárico en el plasma seminal suplementado, se relacionan con una menor calidad seminal postdescongelación. Un nivel bajo de proteínas totales y ácido esteárico, así como un nivel medio de ambas vitaminas en el plasma seminal suplementado, favorecen en términos generales, la movilidad y la integridad de membrana post-descongelación de los espermatozoides. De acuerdo a lo anterior, se concluye que la composición del plasma seminal suplementado para la congelación de semen de asno, es determinante en la calidad espermática post-descongelación.

\section{LITERATURA CITADA}

1 Oliveira RR, Rates DM, Pugliesi G, Ker PG, Arruda RP, Moraes EA, Carvalho GR. Use of cholesterol-loaded cyclodextrin in donkey semen cryopreservation improves sperm viability but results in low fertility in mares. Reprod Domest Anim 2014;49:845-850.

2. Ortiz I, Dorado J, Ramírez L, Morrell JM, Acha D, Urbano M, et al. Effect of single layer centrifugation using Androcoll-E-Large on the sperm quality parameters of cooled-stored donkey semen doses. Anim 2014;8(2):308-315.

3. Carver DA, Ball BA. Lipase activity in stallion seminal plasma and effect of lipase on stallion spermatozoa during storage at $5^{\circ} \mathrm{C}$. Theriogenology 2002;58:1587-95.

4. Contri A, Alessia G, Domenico R, Michele P, Sfirro M, Carluccio A. Effect of sperm concentration on characteristics of frozen-thawed semen in donkeys. Anim Reprod Sci 2012;136:74-80.

5. Jasko DJ, Moran DM, Farlin ME, Squires EL. Effect of seminal plasma dilution or removal on spermatozoal motion characteristics of cooled stallion semen. Theriogenology 1991;35:1059-1068.

6. Miró J, Taberner E, Rivera M, Peña A, Medrano A, Rigau T, Peñalba A. Effects of dilution and centrifugation on the survival of spermatozoa and the structure of motile sperm cell subpopulations in refrigerated Catalonian donkey semen. Theriogenology 2009;72:1017-1022.

7. Kareskoski M, Katila T. Components of stallion seminal plasma and effects of seminal plasma on sperm longevity. Anim Reprod Sci 2008;107:249-256.
8. Rodriguez-Martinez H, Kvist U, Ernerudh J, Sanz L, Calvete JJ. Seminal plasma proteins: what role do they play? Am J Reprod Immunol 2011;66:11-22.

9. Waheed M, El-Bahr SM, Al-Haider AK. Influence of seminal plasma antioxidants and osteopontin on fertility of the Arabian horse. J Equine Vet Sci 2013;33:705-709.

10. Bradford MM. A rapid and sensitive method for the quantitation of microgram quantities of protein utilizing the principle of protein-dye binding. Ann Biochem 1976;72(1-2):248-254.

11. Novakova L, Solich P, Solichova D. HPLC methods for simultaneous determination of ascorbic and dehydroascorbic acids. Trend Annn Chem 2008;27:942-958.

12. Wagner $\mathrm{KH}$, Wotruba $\mathrm{F}$, Elmadfa I. Antioxidative potential of tocotrienols and tocopherols in coconut fat at different oxidation temperatures. Eur J Lipid Sci Tech 2001;103:746-751.

13. Zapata-Luján A, Cogollo-Pacheco A, Rojano BA. Potencial nutracéutico del aceite de la almendra de choibá o almendro de montaña (Dipteryx oleifera Benth). Rev Cubana Plant Med 2013;18(3):368-380.

14. Bustamante I, Pederzolli C, Sgaravatti A, Gregory R, Dutra C, Jobim M, Mattos RC. Skim milk-egg yolk based semen extender compensates for non-enzymatic antioxidant activity loss during equine semen cryopreservation. Anim Reprod Sci 2009;6:392-399.

15. Restrepo G, Ocampo D, Velásquez A. Evaluación de la movilidad del semen criopreservado de caballos criollo colombiano por un sistema analizador de clase. Rev UDCA Act\&Div Cient 2013;16(2):445-450.

16. Gamboa S, Rodrigues A, Henriques L, Batista C, Ramalho-Santos J. Seasonal functional relevance of sperm characteristics in equine spermatozoa. Theriogenology 2010;73(7):950-958.

17. Barth A, Oko R. Abnormal morphology of bovine spermatozoa. 1 ed. Iowa, USA: Iowa State University Press; 1989.

18. Neild D, Chaves G, Flores M, Mora N, Beconi M, Aguero A. Hypoosmotic test in equine spermatozoa. Theriogenology 1999;51:721-727.

19. Ramires-Neto C, Monteiro GA, Fatima-Soares R, Cesar-Pedrazzi R, Dell'aqua JA, Papa FO, Alvarenga MA. Effect of removing seminal plasma using a sperm filter on the viability of refrigerated stallion semen. J Equine Vet Sci 2013;33:40-43.

20. Leeb T, Sieme H, Topfer-Petersen E. Genetic markers for stallion fertility - lessons from humans and mice. Anim Reprod Sci 2005;89:21-29.

21. Hamann H, Jude R, Sieme H, Mertens U, Topfer-Petersen E, Distl $\mathrm{O}$, Leeb $\mathrm{T}$. A polymorphism within the equine CRISP3 gene is associated with stallion fertility in Hanoverian warmblood horses. Anim Genet 2007;38:259-264.

22. Jobim MI, Treina C, Zirklerb H, Gregorya RM, Siemec H, Mattos RC. Two-dimensional polyacrylamide gel electrophoresis of equine seminal plasma proteins and their relation with semen freezability. Theriogenology 2011;76:765-771.

23. Begley A, Quinn P. Decapacitation factors in semen. Clin Reprod Fértil 1982;1:167-175.

24. Snow D, Frigg M. Bioavailability of ascorbic acid in horses. J Vet Pharmacol Ther 1990:13;393-403.

25. Williams C, Carlucci S. Oral vitamin E supplementation on oxidative stress, vitamin and antioxidant status in intensely exercised horses. Equine Vet J 2006;36:617-621. 
26. Carvera DA, Ball BA. Lipase activity in stallion seminal plasma and the effect of lipase on stallion spermatozoa during storage at $5^{\circ} \mathrm{C}$. Theriogenology 2002;58:1587-1595.

27. Morrell JM, Georgakas A, Lundeheim N, Nash D, Davies-Morel MC, Johannisson A. Effect of heterologous and homologous seminal plasma on stallion sperm quality. Theriogenology 2014;82:176-183.

28. Restrepo G, Usuga A, Montoya J, Duque J, Zapata K, Rojano B. Influencia de la concentración de vitamina $C$ sobre la calidad del semen equino criopreservado. Congreso Panamericano de la Ciencias Veterinarias. Habana, Cuba. 2014:6-9.

29. Guasti PN, Souza FF, Scott C, Hartwig FP, Papa MP, Dos Santos GAM, et al. Protein content of equine seminal plasma and sperm plasma membrane in subfertile stallions. J Equine Vet Sci 2014;34:84-85.

30. Carluccio A, Panzani S, Contri A, Bronzo V, Robbe D, Veronesi M. Influence of season on testicular morphometry and semen characteristics in Martina Franca jackasses. Theriogenology 2013;79:502-507.

31. Dorado J, Acha D, Gálvez M, Ortiz I, Carrasco J, Díaz B, GómezArrones V, Calero-Carretero R, Hidalgo M. Sperm motility patterns in Andalusian donkey (Equus asinus) semen: Effects of body weight, age, and semen quality. Theriogenology 2013;79:1100-1109.

32. Dowsett $K$, Knott $L$. The influence of age and breed on stallion semen. Theriogenology 1996;46(3):397-412.

33. Earle KE. Antioxidant nutrients: their role in a healthy diet. Vet Int 2000;12(1):2-7.

34. Loomis P, Graham J. Commercial semen freezing: Individual male variation in cryosurvival and the response of stallion sperm to customized freezing protocols. Anim Reprod Sci 2008;105(1-2):119-128.

35. Burnaugh L, Sabeur K, Ball B. Generation of superoxide anion by equine spermatozoa as detected by dihydroethidium. Theriogenology 2007;67(3):580-589.

36. Pal Y, Dedar R, Ravi S, Legha R, Gupta A, Singh R. Effect of dietary antioxidant supplementation on fresh semen quality in indigenous jacks. Indian Vet J 2013;90(3):135-136.
37. Yousefian I, Zare-Shahneh A, Zhandi M. The effect of coenzyme q10 and a-tocopherol in skim milk-based extender for preservation of caspian stallion semen in cool condition. J Equine Vet Sci 2014;34:949-954.

38. Vasconcelos JS, Chaveiro A, Gois B, Moreira da silva F. Effects of atocopherol and ascorbic acid on equine semen quality after cryopreservation. J Equine Vet 2013;33:787-793.

39. Almeida J, Ball B. Effect of a-tocopherol and tocopherol succinate on lipid peroxidation in equine spermatozoa. Anim Reprod Sci 2005;87(3-4):321-337.

40. Carocho M, Ferreira IC. A review on antioxidants, prooxidants and related controversy: natural and synthetic compounds, screening and analysis methodologies and future perspectives. Food Chem Toxicol 2013;(51):15-25.

41. Morte M, Rodrigues A, Soares D, Rodrigues A, Gamboa S, RamalhoSantos J. The quantification of lipid and protein oxidation in stallion spermatozoa and seminal plasma: Seasonal distinctions and correlations with DNA strand breaks, classical seminal parameters and stallion fertility. Anim Reprod Sci 2008;106(1-2):36-47.

42. Chow P, White I, Pickett B. Stallion sperm and seminal plasma phospholipids and glycerylphosphorylcholine. Anim Reprod Sci 1986;11:207-213.

43. Macías-García B, González-Fernández L, Ortega-Ferrusola C, Salazar-Sandoval C, Morillo-Rodríguez A, Rodríguez-Martínez $\mathrm{H}$, et al. Membrane lipids of the stallion spermatozoon in relation to sperm quality and susceptibility to lipid peroxidation. Reprod Domest Anim 2011;46:141-148.

44. Martínez-Soto J, Landeras J. Gadea J. Spermatozoa and seminal plasma fatty acids as predictors of cryopreservation success. Andrology 2013;1:365-375.

45. Macías-García B, González-Fernández L, Ortega-Ferrusola C, Morillo-Rodríguez A, Gallardo-Bolaños J, Rodriguez-Martinez J, et $a$ al. Fatty acids and plasmalogens of the phospholipids of the sperm membranes and their relation with the post-thaw quality of stallion spermatozoa. Theriogenology 2011;75:811-818. 